\title{
Human thymic MR1-restricted MAIT cells are innate pathogen-reactive effectors that adapt following thymic egress
}

\author{
MC Gold ${ }^{1,2}$, T Eid ${ }^{1}$, S Smyk-Pearson ${ }^{1}$, Y Eberling ${ }^{1}$, GM Swarbrick $^{3}$, SM Langley $^{4}$, PR Streeter ${ }^{3}$, \\ DA Lewinsohn ${ }^{3,5}$ and DM Lewinsohn ${ }^{1,2,5}$
}

Human mucosal-associated invariant T (MAIT) cells express the semi-invariant T-cell receptor (TCR) V $\alpha$ 7.2 and are restricted by the major histocompatibility complex-lb molecule MR1. While MAIT cells share similarities with other innate T cells, the extent to which MAIT cells are innate and their capacity to adapt is unknown. We evaluated the function of $\mathrm{V} \alpha 7.2^{+} \mathrm{T}$ cells from the thymus, cord blood, and peripheral blood. Although antigen-inexperienced MAIT cells displayed a naïve phenotype, these had intrinsic effector capacity in response to Mycobacterium tuberculosis (Mtb)-infected cells. $\mathrm{V} \alpha 7.2^{+}$effector thymocytes contained signal joint TCR gene excision circles (sjTRECs) suggesting limited replication and thymic origin. In evaluating the capacity of Mtb-reactive MAIT cells to adapt, we found that those from the peripheral blood demonstrated a memory phenotype and had undergone substantial expansion, suggesting that they responded to antigenic stimulation. MAIT cells, an evolutionarily conserved T-cell subset that detects a variety of intracellular infections, share features of innate and adaptive immunity.

\section{INTRODUCTION}

The immune recognition of intracellular infection plays a critical role in the host response to both viral and bacterial infection. While innate mechanisms, such as those exemplified by Toll-like receptor and NOD-like receptor family members, have evolved to detect directly the presence of microbial products, these mechanisms are often insufficient in the ultimate containment of intracellular infection. In contrast, cytolytic CD8 ${ }^{+}$ $\mathrm{T}$ cells, by virtue of sampling the intracellular environment via major histocompatibility complex (MHC)-Ia molecules, can play a definitive role in the recognition and ultimate containment of these infections.

MHC-Ia-restricted CD8 ${ }^{+} \mathrm{T}$ cells use a broad array of T-cell receptors (TCRs) to discriminate among MHC molecules bound to a vast array of peptide ligands. Adaptively acquired $\mathrm{CD} 8{ }^{+} \mathrm{T}$ cells have the advantages of specificity and longevity. However, the transition from low-frequency naïve $\mathrm{T}$ cells to highfrequency effector cells can take several days, allowing intracellular pathogens, many of which employ evasion strategies, to establish life-long residency and escape detection and elimination by host immune cells. In this regard, work performed on the recognition of the intracellular pathogen Mycobacterium tuberculosis $(\mathrm{Mtb})$ is illustrative. Adaptively acquired $\mathrm{CD} 4^{+}$ and $\mathrm{CD} 8^{+} \mathrm{T}$ cells are critical for the containment of $\mathrm{Mtb}$, and others and we have found that $\mathrm{CD} 8^{+} \mathrm{T}$ cells reflect the presence and abundance of this pathogen. ${ }^{1-4}$ However, the acquisition of adaptive T-cell immunity in tuberculosis (TB) is delayed relative to that seen in viral infection, and hence provides a window of opportunity for mycobacterial persistence. ${ }^{1,5,6}$

In contrast, innate $\mathrm{T}$ cells operate through the coordinated use of a limited family of TCRs in conjunction with restriction by MHC-Ib molecules that have limited polymorphism. While MHC-Ia-restricted T cells acquire effector function in the periphery following antigenic stimulation, innate T-cell effector function is acquired in the thymus in the absence of exogenous antigenic exposure. This results in the ability of innate $\mathrm{T}$ cells to respond rapidly to intracellular infection, thereby controlling the pathogen as well as facilitating the acquisition of

\footnotetext{
${ }_{1}^{1}$ Pulmonary and Critical Care Medicine, Oregon Health and Science University, Portland, Oregon, USA. Portland VA Medical Center, Portland, Oregon, USA.

${ }^{3}$ Pediatrics, Oregon Health and Science University, Portland, Oregon, USA. Doernbecher Cardiothoracic Surgery, Doernbecher Children's Hospital, Portland,

Oregon, USA. 5 Molecular Microbiology and Immunology, Oregon Health and Science University, Portland, Oregon, USA. Correspondence: MC Gold (goldm@ohsu.edu)

or DM Lewinsohn (lewinsod@ohsu.edu)

Received 23 January 2012; accepted 1 May 2012; published online 13 June 2012. doi: 10.1038/mi.2012.45
} 
effective immunity. In the mouse, $\mathrm{T}$ cells restricted by the MHC$\mathrm{Ib}$ molecule $\mathrm{H} 2-\mathrm{M} 3^{7,8}$ are present in the thymus. Similarly CD1d-restricted invariant natural killer T cells (iNKT) cells ${ }^{9}$ are present in the mouse and human thymus. ${ }^{10,11}$ In addition, we have previously found that the human thymus contains Mtbreactive $\mathrm{CD} 8^{+} \mathrm{T}$ cells of unknown restriction specificity. ${ }^{12}$

MR1-restricted mucosal-associated invariant T (MAIT) cells share a number of characteristics that are associated with innate T cells. Human MAIT cells express a semi-invariant TCR (in humans $\mathrm{V} \alpha 7.2$ ), ${ }^{13,14}$ are restricted by the monomorphic MHC-Ib molecule MR1, and are selected in the thymus on a hematopoietic cell type that remains to be defined. ${ }^{14,15}$ MR1 is the most evolutionarily conserved MHC-like molecule among mammals. ${ }^{16}$ MR1 is predicted to be structurally similar to classical MHC-Ia molecules that have antigen presentation function. ${ }^{17}$

Although the identity of the MR1 ligand(s) is unknown, it is clear that MAIT cells are capable of detecting host cells infected with a variety of microbes, including $\mathrm{Gram}^{+}$and $\mathrm{Gram}^{-}$bacteria, fungi, and mycobacteria in an MR1-restricted manner. ${ }^{18,19}$ Thus, the combination of the semi-invariant TCR and the monomorphic MR1 molecule and ligand must in concert allow for the detection of this wide array of microbes. As such, we believe the use of Mtb, as a model antigen to stimulate MAIT cells, is generally applicable to the study of MAIT cells.

Nonetheless, whether or not human antigen-inexperienced MR1-restricted T cells have innate functional capacity is unknown. Furthermore, increasing evidence suggests that, like other T-cell subsets, MAIT cells can adapt following thymic egress. Following microbial colonization, mouse MAIT cells expand, ${ }^{14,19}$ suggesting activation and possibly differentiation as a consequence of peripheral stimulation. In humans, MAIT cells are decreased in the blood of patients with active TB or other bacterial pneumonias. ${ }^{18,19}$ Whether or not these changes are associated with long-term adaptation is not known.

To address the hypothesis that human MAIT cells are innate, we assessed the capacity of antigen-inexperienced MAIT cells to perform as direct ex vivo effectors. Here, we have used the model pathogen Mtb to compare and contrast the function and phenotype of human, antigen-inexperienced, thymic-resident MAIT cells with those in the cord blood and in the peripheral circulation. We found that Mtb-reactive Va7.2 ${ }^{+} \mathrm{CD} 8{ }^{+}$MAIT cells were capable of short-term cytokine production in the thymus and cord blood of all donors tested. Moreover, we found that functional MAIT thymocytes contained high levels of signal joint TCR gene excision circles (sjTRECs), indicating that these cells had undergone minimal replication. Because MR1 is necessary for the intrathymic selection of MAIT cells, we sought to determine the cellular source of this ligand. Robust expression of MR1 was present exclusively on a subset of $\mathrm{CD}^{+}$thymocytes, suggesting that these $\mathrm{T}$ cells could serve to select MAIT cells in the human thymus. In evaluating the adaptive capacity of MAIT cells, we found that in contrast to the naïve phenotype of effector MAIT cells from the thymus and cord blood, the peripheral blood Mtb-reactive MAIT cells expanded and expressed a memory T-cell phenotype, suggesting that MAIT cells are capable of maturation in response to peripheral antigenic stimulation. From these data, we conclude that human MAIT cells can be considered both innately and adaptively acquired.

\section{RESULTS}

We previously demonstrated that MR1-restricted Mtb-reactive MAIT cells from the peripheral blood are present in the $\mathrm{CD} 8^{+}$, but not in the $\mathrm{CD}^{-}{ }^{-} \mathrm{CD} 8^{-}$(double negative (DN)) T-cell subset. ${ }^{18}$ Consequently, we sought to evaluate the requirement for CD8 co-receptor engagement for MAIT cell recognition of Mtb-infected antigen-presenting cells. Figure 1 shows that CD8 blockade abrogated the ability of three independently derived $\mathrm{CD}^{+}{ }^{+}$Mtb-reactive MR1-restricted clones ${ }^{18}$ to detect infected cells. Similar results were obtained using Mtb-infected epithelial cells as antigen-presenting cells (not shown). In contrast, CD8 blockade had no effect on an Mtb-specific MHC-II-restricted clone (Figure 1). These data demonstrate that CD8 is required for the activation of these MAIT cells.

To establish the relationship between prior antigenic exposure and the prevalence and phenotype and MR1-restricted MAIT cells, we performed flow cytometric analysis of MAIT cells derived from human thymus $(n=6)$, cord blood $(n=4)$, and peripheral blood $(n=6)$. We first determined the total frequency of $\mathrm{CD}^{+} \mathrm{V} \alpha 7.2^{+}$cells (Figure 2b). $\mathrm{CD} 8^{+} \mathrm{Va} 7.2^{+}$cells were detectable from all tissue sources and from all donors (Figure 2a and $\mathbf{b})$. The frequencies of $\mathrm{CD}^{+} \mathrm{T}$ cells that expressed $\mathrm{V} \alpha 7.2$ in the thymus ( $n=6$; mean: 1.8 ; s.e.m.: 0.6$)$ were lower compared to those in the cord blood $(n=4$; mean 4.2; s.e.m.: $1 ; P=0.05)$ and peripheral blood ( $n=6$; mean: 4.3 ; s.e.m.: 1 ; $P=0.04)$.

However, antibody detection of $\mathrm{V} \alpha 7.2^{+} \mathrm{T}$ cells alone does not allow for the identification of functional MR1-restricted MAIT cells. The anti-Va7.2 antibody that was published previously (clone $3 \mathrm{C} 10)^{15}$ as well as the recently developed anti-V $\alpha 7.2$ antibody (clone OF-5A12) (Supplementary Figures S1 and S2 online) label all $\mathrm{T}$ cells that express the Va7.2 TCR including

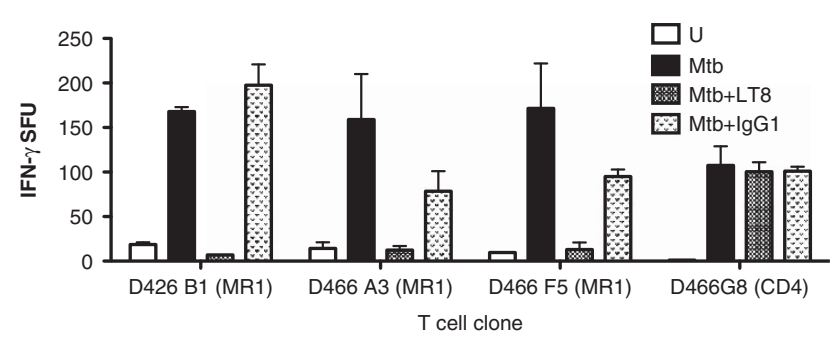

Figure 1 CD8 co-receptor blockade prevents the detection of Mycobacterium tuberculosis (Mtb)-infected dendritic cells (DCs) by human MR1-restricted Mtb-reactive CD8 ${ }^{+}$T-cell clones. D466 DCs were left uninfected $(U)$ or infected with Mtb (multiplicity of infection (MOI) 30) overnight and incubated (10,000 per well) with Mtb-reactive MR1-restricted CD8 ${ }^{+}$T-cell clones (D426B1, D466A3, D466F5) (10,000 per well) or the Mtb-specific CD4 + T-cell clone (D466G8) (5,000 per well) in the presence of CD8 blocking antibody LT8 or control mslgG1 antibody $\left(2.5 \mu \mathrm{g} \mathrm{ml}^{-1}\right.$ each). Interferon (IFN)- $\gamma$ production was detected the following day by ELISPOT. Each bar and error bars represent the mean and s.e.m., respectively, of IFN- $\gamma$ spot-forming units (SFUs) from duplicate wells. Similar results were obtained in three independent experiments. 


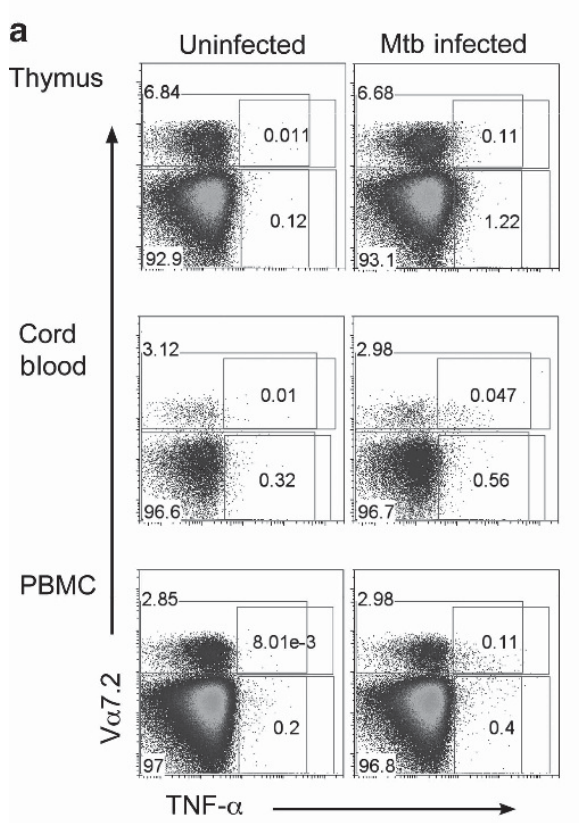

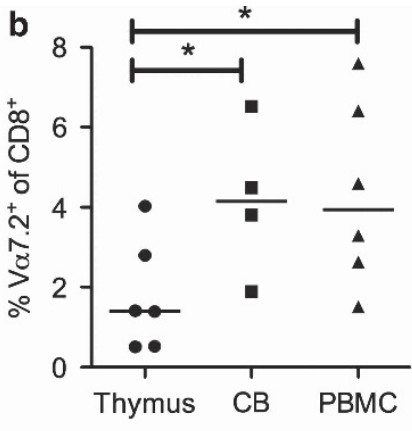
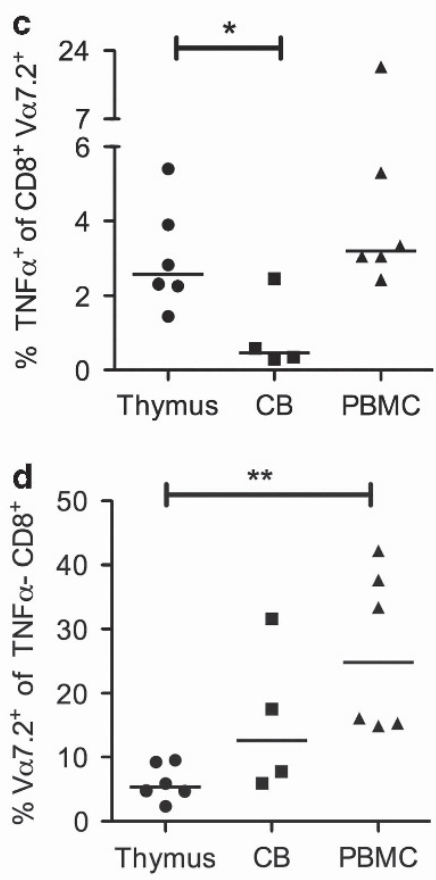
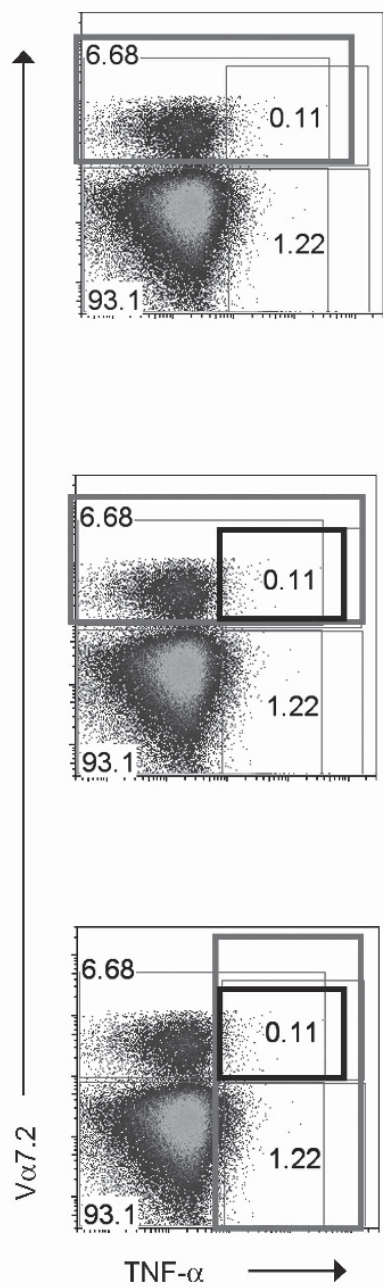

Figure 2 Human mucosal-associated invariant T (MAIT) cells from the thymus and cord blood have innate ex vivo effector capacity. CD4-depeletd T cells from the thymus, cord blood, and peripheral blood (500,000 per well) were incubated with stimulator A549 cells left uninfected or infected with Mycobacterium tuberculosis (Mtb) $\left(1.5 \mathrm{e} 6 / 2 \mathrm{~cm}^{2}\right.$ well) and tested for the intracellular production of tumor necrosis factor- $\alpha$ (TNF- $\alpha$ ) as described in Methods. Briefly, after overnight incubation, a protein transport inhibitor was added for the final $6 \mathrm{~h}$ of the assay, after which cells were surface-stained for expression of the V $\alpha 7.2$ T-cell receptor (TCR) and CD8, and thereafter fixed and permeabilized before staining for intracellular TNF- $\alpha$. As TNF- $\alpha^{+}$ $\mathrm{V} \alpha 7.2^{+}$is not present in the CD8 ${ }^{-}$subset, all frequencies were determined from CD8 ${ }^{+}$gated events only. (a) Dot-plot analyses from the intracellular cytokine staining assay demonstrating representative responses to uninfected A549 (left column) or Mtb-infected A549 (right column) from an individual thymus, cord blood, and adult donor. Cells are gated on CD8 ${ }^{+}$events. The $x$ axis represents TNF- $\alpha$; the $y$ axis represents $V \alpha 7.2$. Numbers represent the frequency of $\mathrm{CD}^{+}$events within each gate. (b) Frequencies of $\mathrm{V} \alpha 7.2^{+} \mathrm{CD} 8^{+}$cells from the thymus $(n=6$; mean: 1.8; s.e.m.: 0.6), cord blood ( $n=4$; mean 4.2; s.e.m.: 1.0), and peripheral blood ( $n=6$; mean: 4.3; s.e.m.: 1.0). Results for each donor were derived using the frequencies of cells derived from the red gate (right panel). Significant differences were detected between the thymus and cord blood $(P=0.05)$ and the thymus and peripheral blood $(P=0.04)$. (c) Frequencies of $\mathrm{V} \alpha 7.2^{+} \mathrm{CD} 8^{+}$cells that produced TNF- $\alpha$ in response to Mtb-infected A549 cells. Thymus ( $n=6$; mean: 3.02; s.e.m.: 0.58), cord blood ( $n=4$; mean 0.92 ; s.e.m.: 0.52), and peripheral blood ( $n=6$; mean: 6.20 ; s.e.m.: 2.80). Results for each donor were derived using the percentage of cells in the black gate as a proportion of those in the red gate (right panel). Significant differences were observed between thymus and cord blood $(P=0.04)$. (d) Frequencies of $\mathrm{V} \alpha 7.2^{+}$cells among Mtb-reactive TNF $\alpha^{+} \mathrm{CD} 8^{+}$cells. Thymus $(n=6 ;$ mean: 6.07 ; s.e.m.: 1.14), cord blood ( $n=4$; mean 15.68; s.e.m.: 5.89), and peripheral blood ( $n=6$; mean: 26.60 ; s.e.m.: 5.12$)$. Results for each donor were derived using the percentage of cells in the black gate as a proportion of those in the red gate (right panel). Significant differences were observed between thymus and peripheral blood $(P=0.002)$. The unpaired two-tailed, $t$-test was used to assess significant differences between groups. ${ }^{*} P$-value $<0.05 ;{ }^{* *} P$-value $<0.01$.

those that are MHC-Ia-restricted. Therefore, to establish the frequency and phenotype of functional MR1-restricted Mtb-reactive Va7.2 ${ }^{+}$cells ex vivo, we employ an assay that uses Mtb-infected A549 epithelial cells as stimulators. ${ }^{18}$

We evaluated the frequency of Mtb-reactive MAIT cells from the thymus, cord blood, and peripheral blood using
CD4-depleted T cells that contain both $\mathrm{CD}^{+}$and DN T cells. CD4-depleted $\mathrm{T}$ cells from all donors were incubated with Mtbinfected A549 cells. Effector MAIT cells were enumerated using intracellular staining for tumor necrosis factor- $\alpha$ (TNF- $\alpha$ ) in conjunction with cell surface staining for the V $\alpha 7.2$ TCR that defines MAIT cells using OF-5A12 (Supplementary Figures S1 and S2). 
Representative flow cytometric plots from the thymus, cord blood, and peripheral blood are depicted in Figure 2a, demonstrating that uninfected A549 cells do not elicit nonspecific responses.

Consistent with our prior report, ${ }^{18}$ we found that pathogenreactive MAIT cells from the thymus and cord blood, in addition to peripheral blood, were present in the $\mathrm{CD} 8{ }^{+}$population and were MR1-restricted (Supplementary Figure S3 and data not shown). On average, Mtb-reactive TNF- $\alpha^{+}$MAIT cells comprised $3.0 \%$ of $\mathrm{V} \alpha 7.2^{+} \mathrm{CD} 8{ }^{+} \mathrm{T}$ cells from the thymus, $0.92 \%$ from the cord blood, and $6.2 \%$ from the peripheral blood (Figure 2c). Mtb-reactive MAIT cells from antigen-inexperienced sources were detected from all donors at frequencies substantially higher than those expected from naïve conventional CD8 ${ }^{+}$T cells. $^{20}$

Although our data clearly established the presence of Mtbreactive MAIT cells in the thymus, it was possible that these cells were derived from a peripheral antigen-experienced population. ${ }^{21}$ To confirm that MAIT cells from the thymus and cord blood were naïve, we quantified the content of sjTRECs in V $\alpha 7.2^{+} \mathrm{T}$ cells. sjTRECs are circular DNA products resulting from intrathymic $V(D) J$ TCR gene recombination. ${ }^{22}$ As sjTRECs do not replicate during mitosis, they are diluted during cell division. Consequently, sjTREC levels are highest in thymocytes that have recently rearranged their TCR, lower in peripheral naïve $\mathrm{T}$ cells, and undetectable in previously activated $\mathrm{T}$ cells that have by definition undergone extensive division. As such, the prevalence of sjTRECs can be used to assess the replicative history of a T-cell population.

Using quantitative polymerase chain reaction, we estimated sjTREC content from live, fluorescence-activated cell sorting (FACS)-sorted CD8 single-positive (SP) V $\alpha 7.2^{+}$or Va7.2- from the thymus, cord, and peripheral blood. ${ }^{23}$ As expected, sjTREC levels were undetectable in $\mathrm{V} \alpha 7.2^{+}$and $\mathrm{V} \alpha 7.2^{-} \mathrm{CD} 8^{+} \mathrm{T}$ cells isolated from adult peripheral blood that contain predominantly antigen-experienced T cells (Figure 3a). We detected similar levels of sjTRECs from both V $\alpha 7.2^{+}$and $\mathrm{V} \alpha 7.2^{-}$, cord bloodderived $\mathrm{CD} 8^{+}$cells (Figure 3a). Notably, Va7.2 ${ }^{+}$and Va7.2CD8 SP thymocytes had levels of sjTRECs similar to total CD8 SP thymocytes, indicating that these cells had not undergone extensive cell division (Figure 3a). Nonetheless, sjTREC levels from V $\alpha 7.2^{+}$CD8 SP thymocytes were significantly $(P=0.02)$ reduced compared to those from the Va7.2- CD8 SP thymocyte subset (Figure 3a and $\mathbf{b}$ ).

One possible explanation for the reduced sjTREC content in the V $\alpha 7.2^{+}$CD8 SP thymocytes was that these represented a heterogeneous population of cells containing both naïve and antigen-experienced T cells. Antigen-experienced T cells from the periphery can recirculate back to the thymus. ${ }^{21}$ Such previously activated $\mathrm{T}$ cells could perform as short-term effectors, but would have undetectable sjTRECs. In contrast, we presumed that naïve $\mathrm{V} \alpha 7.2^{+} \mathrm{CD} 8 \mathrm{SP}$ thymocytes with innate effector capacity would retain quantifiable sjTRECs.

To evaluate the sjTREC content from live functional $\mathrm{V} \alpha 7.2^{+}$ CD8 SP effectors from the thymus, we performed a short-term $(2 \mathrm{~h})$ stimulation with phorbol myristate acetate and ionomycin.
We previously showed that Mtb-reactive thymocytes express granzyme, a component of the granule exocytosis pathway. ${ }^{12}$ To be able to FACS-isolate live cells with the capacity to degranulate, we utilized the CD107a/LAMP assay ${ }^{24}$ after confirming concordance between CD107a/LAMP expression and TNF- $\alpha$ in Mtb-reactive thymocytes (not shown). Figure 3c shows a representative staining of CD107a/LAMP by V $\alpha 7.2^{+}$and Va7.2- CD8 SP thymocytes in response to phorbol myristate acetate/ionomycin stimulation (right panel). We FACS-sorted four populations of live CD8 SP thymocytes $(n=4)$ based on V $\alpha 7.2$ and LAMP expression and sjTRECs were assessed. We found that sjTRECs were detectable from all subsets regardless of their ability to degranulate (Figure 3d), indicating that functional MAIT cells had not undergone extensive proliferation and were unlikely to be derived from the peripheral blood (Figure 3d). Equivalent sjTREC content was present in LAMP ${ }^{+}$ and LAMP ${ }^{-} \mathrm{V} \alpha 7.2^{-} \mathrm{CD} 8 \mathrm{SP}$ subsets $(P=0.61)$. However, $\mathrm{V} 7.2^{+} \mathrm{LAMP}^{+}$cells had a $32 \%$ reduction in sjTRECs compared to their LAMP $^{-}$counterparts $(P=0.02 ; n=4)$. In combination, these data suggest that as a population, functional MAIT thymocytes are not derived from the peripheral blood, but undergo additional intrathymic cell division.

We next addressed phenotypic changes that MAIT cells undergo once they exit the thymus and encounter peripheral antigen(s). A complex relationship exists between microbes and MAIT cells. MAIT cells are undetectable in the gut lamina propria of germ-free mice, but can be induced to expand after microbial colonization. ${ }^{14,19}$ Furthermore, MAIT cells predominate in tissue sites, including the lung, ${ }^{18}$ the liver, ${ }^{25}$ and the gut lamina propria. ${ }^{14}$ Taken together, these findings suggest a close interrelationship between MAIT cells and microbes located in the mucosa. Therefore, to determine if MAIT cells adapt to their peripheral environment, we compared the maturation state of Mtb-reactive MAIT cells in the thymus, cord blood, and peripheral blood. Cell surface expression of CD45RA, CCR7, and CD62L expression were used to define naïve, effector, and memory states of MAIT cells.

The CD45RA isoform on T cells has been used to delineate broadly naïve T cells, while CD45RO expression in conjunction with the absence of CD45RA is associated with antigen experience. In the human thymus, however, the majority of CD8 SP thymocytes express the CD45RO isoform and only $20 \%$ express CD45RA. ${ }^{26,27}$ The upregulation of the CD45RA isoform on CD8 SP thymocytes is thought to coincide with their final maturation step allowing thymic egress. ${ }^{26-28}$ In evaluating thymocytes, we found identical expression of CD45RA on Mtb-reactive MAIT cells, Va7.2 ${ }^{+} \mathrm{CD} 8{ }^{+}$ T cells, and total CD8 SP thymocytes (Figure 4, left column), suggesting that the functional capacity of MAIT cells in the thymus is achieved before final commitment for thymus egress.

The cord blood primarily contains naïve $\mathrm{T}$ cells that are defined by the expression of the CD45RA isoform. We found no significant differences in the frequencies of CD45RA ${ }^{+}$cells between total CD8 SP, CD8 ${ }^{+} \mathrm{V} \alpha 7.2^{+}$, and Mtb-reactive V $\alpha 7.2^{+}$ $\mathrm{CD}^{+}$cord blood $\mathrm{T}$ cells, further suggesting that functional MAIT cells have a naïve phenotype. In the peripheral blood, total $\mathrm{CD}^{+}{ }^{+} \mathrm{T}$ cells and $\mathrm{V} \alpha 7.2^{+} \mathrm{CD} 8{ }^{+}$cells contained both $\mathrm{CD} 45 \mathrm{RA}{ }^{+}$ 
a

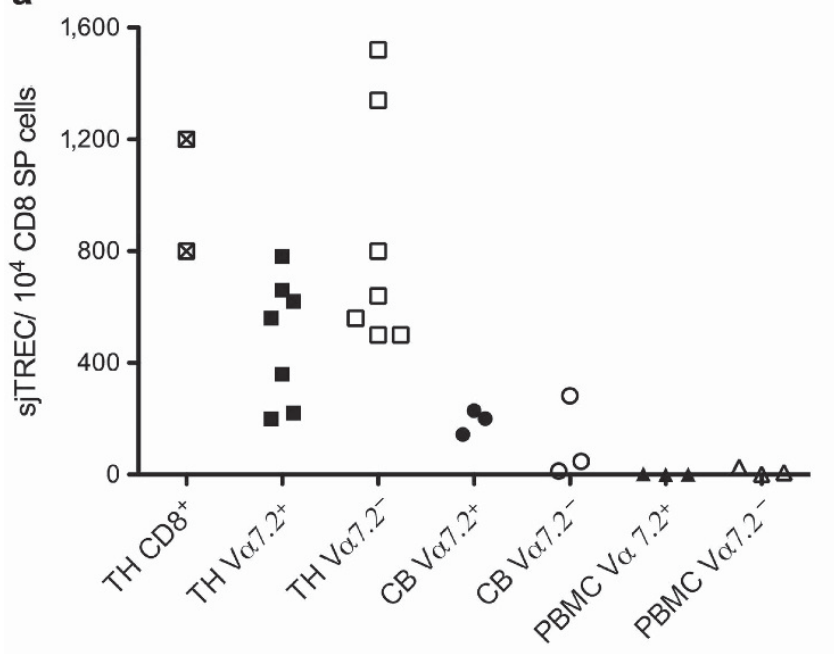

C

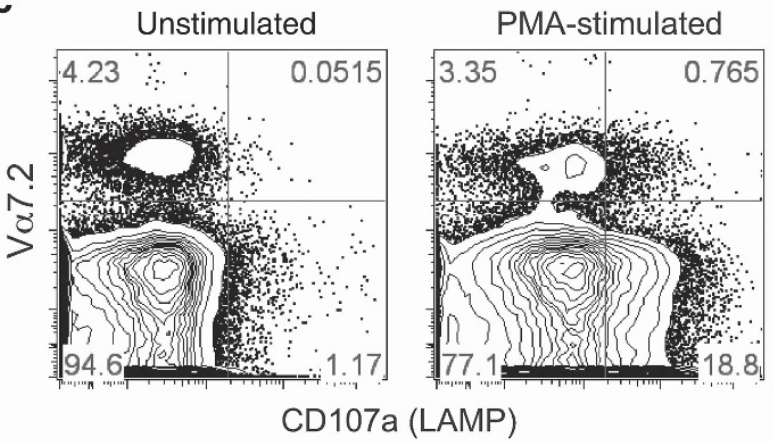

b

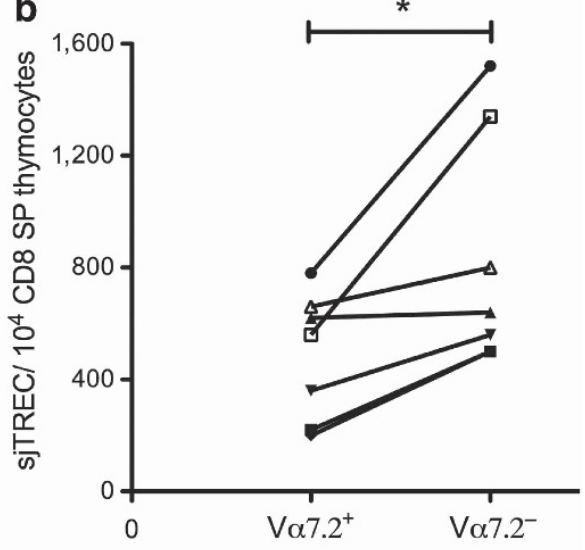

d
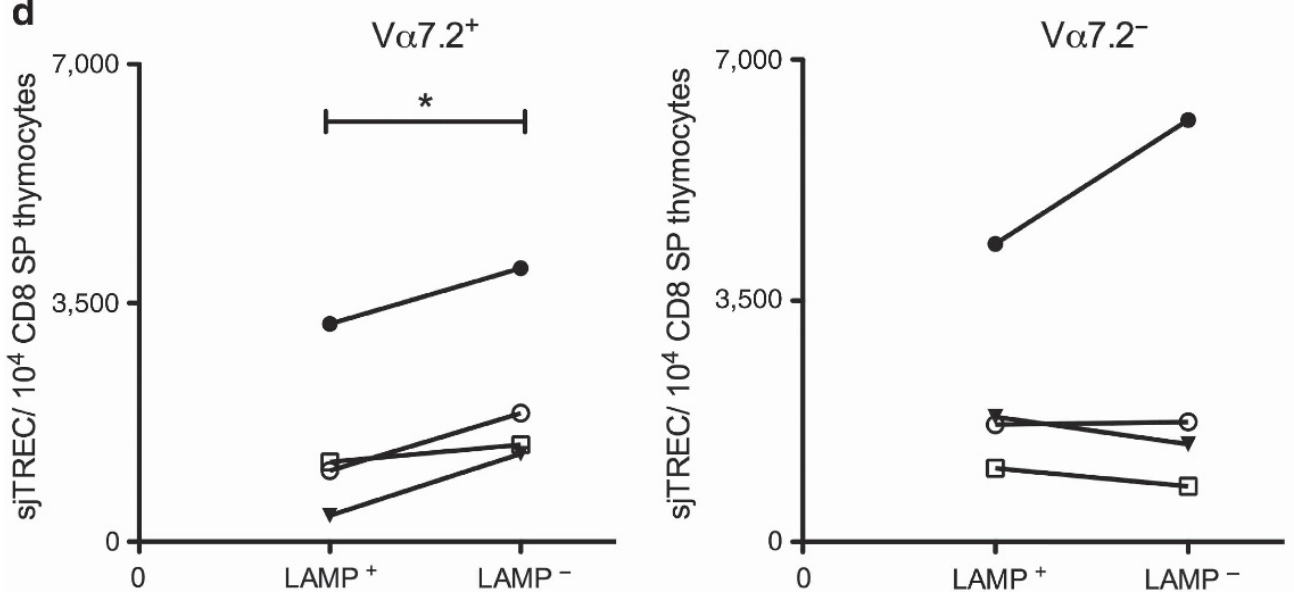

Figure 3 Human $\mathrm{V} \alpha 7.2^{+}$thymocytes and functional mucosal-associated invariant T (MAIT) thymocytes retain signal joint T-cell receptor (TCR) gene excision circles (sjTRECs). (a) sjTREC content per $10^{4}$ cells from the following fluorescence-activated cell sorting (FACS)-sorted subsets; total $\mathrm{CD}^{+}$thymocytes $(n=2)$; paired CD8 ${ }^{+} \mathrm{V} \alpha 7.2^{+}$and $\mathrm{V} \alpha 7.2^{-}$populations from the thymus $(n=7)$, cord blood $(n=3)$, and peripheral blood mononuclear cells (PBMCs) $(n=3)$. (b) Analysis of thymocyte data in (a) demonstrating that CD8 ${ }^{+} \mathrm{V} \alpha 7.2^{+}$thymocytes have significantly less sjTRECs than their paired $\mathrm{CD} 8^{+} \mathrm{V} \alpha 7.2^{-}$thymocytes $(P=0.02)$. (c) Representative dot plot of $\mathrm{V} \alpha 7.2$ and LAMP expression on $\mathrm{CD}^{+} \mathrm{CD}^{-}, \mathrm{PI}^{-}$, and $\gamma \delta \mathrm{TCR}^{-}$thymocytes stimulated without (left) or with (right) phorbol myristate acetate (PMA)/ionomycin for $2 \mathrm{~h}$. (d) sjTREC analysis from FACS-sorted subsets. Significant differences $(P=0.02)$ were observed between the $\mathrm{LAMP}^{+}$and $\mathrm{LAMP}^{-} \mathrm{CD} 8^{+} \mathrm{CD}^{-}, \mathrm{PI}^{-}, \gamma \delta \mathrm{TCR}^{-}$thymocytes that were $\mathrm{V} \alpha 7.2^{+}$but not $\mathrm{V} \alpha 7.2^{-}$. The paired two-tailed, $t$-test was used to assess significant differences between groups. ${ }^{*} P$-value $<0.05$. $\mathrm{PI}$, propidium iodide.

and CD45RA ${ }^{-}$cells. In contrast, Mtb-reactive MAIT cells were uniformly $\mathrm{CD}^{-} 5 \mathrm{RA}^{-}$, a phenotype associated with antigenic stimulation (Figure 4, left column). Furthermore, Va7.2 ${ }^{+} \mathrm{CD} 8^{+}$ cells in the periphery were proportionally expanded within the total non-classical Mtb-reactive $\mathrm{CD} 8^{+} \mathrm{T}$ cells (Figure 2d). These data suggest that once in the periphery, MAIT cells have the capacity to divide and differentiate following exposure to antigenic and/or environmental stimuli. 
Thymus
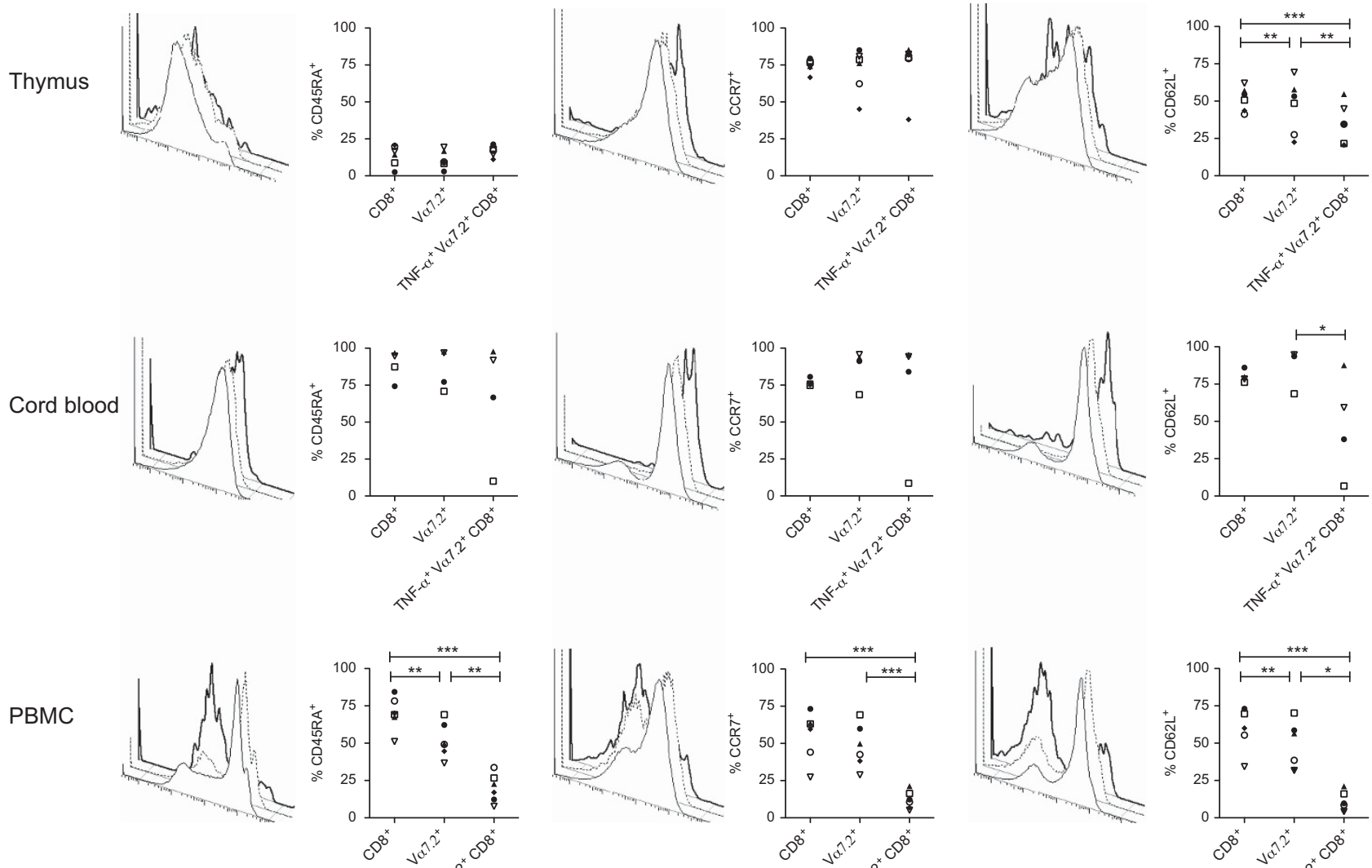

CCR7
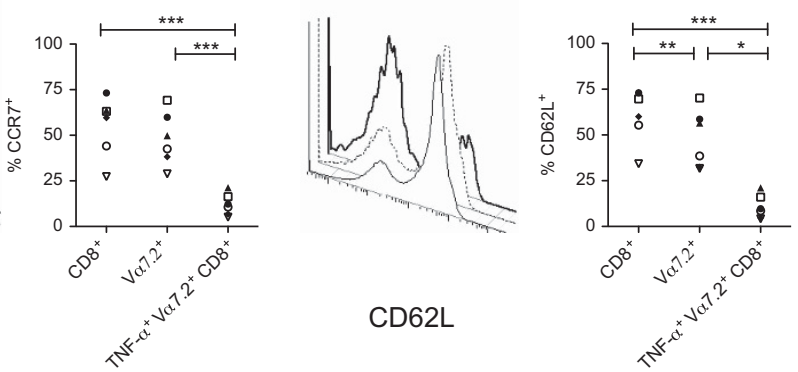

Figure 4 Antigen-inexperienced mucosal-associated invariant T (MAIT) cells are inherently innate effectors with a naïve phenotype, while peripheral blood MAIT cells display a phenotype associated with antigen exposure. The intracellular cytokine staining assay was performed as described in Figure 1. In addition, cells were surface stained with antibodies for CD45RA, CD62L, and CCR7. Frequencies and representative histograms represent expression of CD45RA (left column), CCR7 (middle column), or CD62L (right column) on total CD8 ${ }^{+}$cells (solid line), V $\alpha 7.2^{+} \mathrm{CD} 8^{+}$cells (dashed line), and TNF- $\alpha^{+}$V $\alpha 7.2^{+} \mathrm{CD}^{+}$cells (bold line) from the thymus $(n=6)$, (top row), cord blood $(n=4)$, (middle row), and peripheral blood ( $n=6$ ) (bottom row). Within each graph, frequencies from each donor are represented by the same symbol. The paired two-tailed, $t$-test was used to assess significant differences between groups. ${ }^{\star} P$-value $<0.05 ;{ }^{* \star} P$-value $<0.01 ;{ }^{* \star \star} P$-value $<0.001$. TNF- $\alpha$, tumor necrosis factor- $\alpha$.

To further define their cell surface phenotype, we evaluated the expression of CCR7 and CD62L on MAIT cells. Conventionally, naïve $\mathrm{T}$ cells co-express the chemokine receptor CCR7 and the selectin CD62L, while effector T cells lack surface expression of both molecules. As shown in Figure 4 (middle panel), the majority of Mtb-reactive MAIT cells in the thymus and cord blood expressed CCR7, while those in the peripheral blood completely lacked the expression of CCR7. This dichotomy once again reflected the naive phenotype of Mtb-reactive MAITs from the thymus and cord blood in contrast to the antigenexperienced phenotype in the peripheral blood Mtb-reactive MAIT cells.

However, in contrast to CCR7, we found significant decreases in the frequencies of Mtb-reactive MAIT cells from the thymus and cord blood that expressed the CD62L marker typically present on naïve T cells (Figure 4, right panel). On thymocytes, expression of CD62L is characteristically associated with their imminent egress from the thymus. ${ }^{28,29}$ However, $\mathrm{CD} 8{ }^{+}$mature thymocytes lacking CD62L may preferentially home to nonlymphoid tissues. ${ }^{30}$ In peripheral blood, CD62L expression on
Mtb-reactive MAIT cells was absent consistent with an antigen-experienced phenotype. Thus, the overall phenotype of Mtb-reactive MAIT cells from the thymus and cord blood is consistent with a naïve phenotype, but one that may preferentially allow these cells to home to tissue sites.

As with conventional T cells, iNKT cells and MAIT cells are selected in the thymus in an MHC-dependent manner on CD1d and MR1, respectively. However, unlike conventional $\mathrm{T}$ cells that are selected on thymic epithelial cells, ${ }^{28}$ MHC-Ib-restricted T cells including H2-M3-restricted T cells, ${ }^{8,31}$ iNKT cells, ${ }^{11}$ and MAIT cells ${ }^{15}$ are selected on hematopoietic cells. For thymic selection of iNKT cells, $\mathrm{CD} 4{ }^{+} \mathrm{CD} 8^{+}$double-positive cortical thymocytes that express CD1d are required. ${ }^{11}$ Similarly, MAIT cell selection is thought to occur on a hematopoietic cell subset ${ }^{15}$ that remains to be identified. Given the requirement for MHC in thymic selection, we reasoned that candidate hematopoietic cells that positively select MAIT cells would express MR1.

Although MR1 transcription and translation are thought to be ubiquitous in all cell types, ${ }^{16,32}$ endogenous MR1 cell surface 


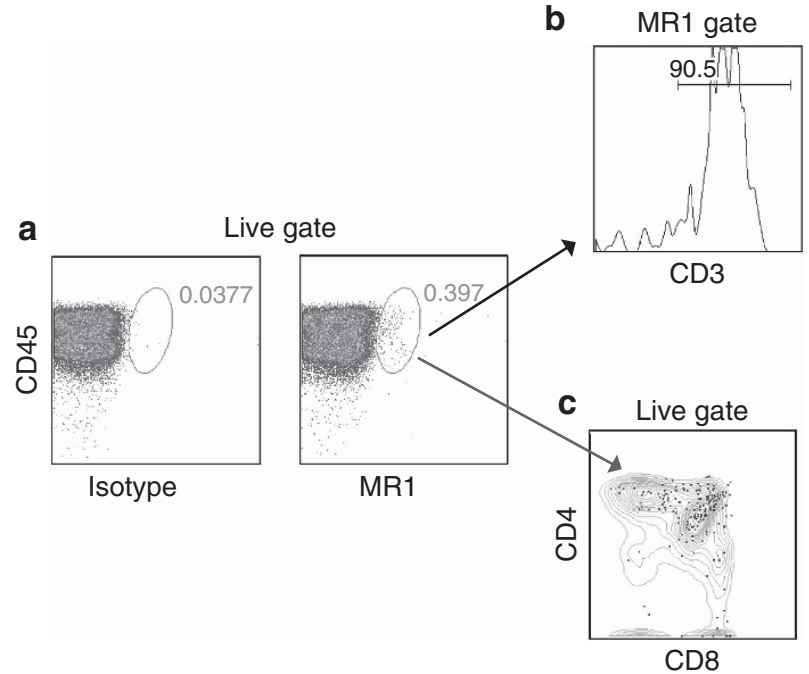

Figure 5 Endogenous MR1 is expressed at high levels on a subset of $\mathrm{CD}_{4} 5^{+} \mathrm{CD}^{+}$thymocytes. Representative analysis of single-cell suspensions of thymocytes $(n=4)$ stained with antibodies for pan-CD45, CD3, CD4, CD8, and MR1 (clone 26.5), and analyzed by flow cytometry. (a) Dot plots of CD45 expression ( $y$ axis) and mslgG2a isotype control or MR1 ( $x$ axis) from live cells. (b) Histogram of CD3 expression on MR1 ${ }^{+}$ cells. (c) Dot plot of CD8 ( $x$ axis) and CD4 expression ( $y$ axis) from total live thymocytes (gray) or MR1-gated cells (red). The mean frequency of $\mathrm{MR} 1{ }^{+}$cells from total live thymocytes $(n=4)$ was $0.37 \%$ (s.e.m.: 0.02 ). Similar results were seen from all for thymocyte donors.

expression has historically been difficult to detect. Modest levels of endogenous MR1 have been detected on mouse cells after the addition of an anti-MR1 antibody that stabilizes cell surface MR1. ${ }^{33}$ In our studies, we detected surface MR1 on epithelial cell line A549 after infection with Mtb. ${ }^{18}$

To determine whether or not endogenous MR1 is expressed in the human thymus, we performed flow cytometric analysis of human thymus single-cell suspensions $(n=4)$. CD45 expression was used to delineate hematopoietic from non-hematopoietic cells. Figure 5 shows a representative analysis demonstrating that MR1 cell surface was only detectable in the $\mathrm{CD} 45^{+}$subset. We did not detect MR1 on CD45- cells from any of the donors $(n=4)$. We also found that MR1 expression was highly restricted to cells expressing the T-cell marker CD3 (Figure 5b). Further analysis of MR1-expressing cells indicated that these were primarily $\mathrm{CD} 4{ }^{+} \mathrm{CD} 8{ }^{+}$double-positive thymocytes. However, $\mathrm{MR}^{+}{ }^{+}$cells could be found in all T-cell subsets except the $\mathrm{CD} 4^{-} \mathrm{CD} 8^{-} \mathrm{DN}$ subset (Figure $5 \mathrm{c}$ ). These results suggest that, as with CD1d-restricted iNKT cells, ${ }^{34}$ an MR1-expressing $\mathrm{T}$ cell could serve as the selecting cell for MAIT cells in the human thymus.

\section{DISCUSSION}

We demonstrate that semi-invariant human MR1-restricted V $\alpha 7.2^{+}$MAIT cells are innately pathogen reactive. First, we demonstrate that a population of $\mathrm{V} \alpha 7.2^{+} \mathrm{T}$ cells from the human thymus and cord blood have the capacity to respond to bacterial infection in the absence of prior antigenic exposure. As such, these cells display functional immediacy, a key feature of innate immunity. We note that the thymuses used in these studies were derived from neonates within days of birth. Thus, it is unlikely that exogenous microbial exposures could account for our results. Furthermore, we found that functional V $\alpha 7.2^{+}$ cells in the thymus contain sjTRECs at levels similar to naïve conventional thymocytes, supporting the hypothesis that these $\mathrm{V} \alpha 7.2^{+} \mathrm{T}$ cells are not simply derived from peripheral blood effectors. Nonetheless, in comparison to CD8 SP V $\alpha 7 .^{-}$ $\mathrm{T}$ cells, those cells expressing Va7.2 had undergone modest but increased intrathymic cell division. This observation suggests that MAIT cells, like iNKT cells, ${ }^{11}$ undergo some cell division in the thymus before release into the periphery. This intrathymic cell division is likely to contribute to readily detectable frequencies of MAIT cells, which in combination with their intrinsic effector function suggests that MAIT cells can contribute to early control of intracellular microbial infection. In mice, MR1dependent control of Klebsiella pneumoniae was demonstrated within the first 4 days of infection. ${ }^{35}$

A distinguishing feature of innate $\mathrm{T}$ cells is the manner in which they are selected in the thymus. Specifically, innate T cells are selected in the thymus on hematopoietic cells in contrast to conventional $\mathrm{T}$ cells that are selected on thymic epithelial cells. T-cell selection by hematopoietic cells has been shown for MHC-Ib-restricted T cells notably $\mathrm{H} 2-\mathrm{M}^{8}$ and $\mathrm{CD} 1 \mathrm{~d}$-restricted T cells. ${ }^{11}$ Like human MAIT cells, both of these cell types can be found as thymic effectors. Furthermore, selection by hematopoietic cells resulted in H2-M3-restricted T cells with an activated phenotype with potent effector function, in contrast to those selected on thymic epithelial cells. ${ }^{31}$ Thus, a correlation exists between hematopoietic cell selection and innate T-cell programming. It is known that MAIT cells are selected in the thymus in an MR1-dependent manner on a hematopoietic cell. ${ }^{14,15}$ Here we show that MR1 is uniquely expressed on a subset of $\mathrm{CD} 3^{+}$ $\mathrm{CD} 45^{+}$hematopoietic T cells. Moreover, MR1 is expressed at relatively high levels on these cells as compared to other cell subsets, both as we have observed (data not shown) and as reported by others. ${ }^{33,36}$ In addition, these data are consistent with those recently published by Chua et al. ${ }^{33}$ in which mouse doublepositive thymocytes were capable of MR1-dependent direct stimulation of MAIT cell hybridomas. We suggest that MR1-restricted innate V $\alpha 7.2^{+} \mathrm{T}$ cells are positively selected in the thymus via endogenous MR1 on this $\mathrm{CD}^{+} \mathrm{MR}^{+}$ thymocyte subset.

Innate $\mathrm{T}$ cells are often enriched in specific tissue sites. For example, iNKT cells are preferentially distributed to the liver in a manner dependent on the transcription factor PLZF. ${ }^{37-39}$ We have observed the enrichment of MR1-restricted Mtbreactive $\mathrm{V} \alpha 7.2^{+} \mathrm{T}$ cells in the lung. ${ }^{18}$ The relative absence of CD62L on the surface of Mtb-reactive thymic-resident MAIT suggests that MAIT cells are inherently programmed to traffic to tissue sites. ${ }^{30}$

The relative expansion of $\mathrm{V} \alpha 7.2^{+} \mathrm{T}$ cells as a proportion of the total Mtb-reactive non-classical CD8 ${ }^{+} \mathrm{T}$-cell response suggests that microbial-reactive MAIT cells have the capacity to adapt to the peripheral environmental signals. In the thymus, MAIT cells accounted for $6 \%$ of the Mtb-reactive non-classical CD8 ${ }^{+}$ 
subset. This proportion increased in the cord blood to $16 \%$ and ultimately reached $27 \%$ in the periphery. These findings are similar to those previously published in ruminants and mice. Neonatal ruminants have detectable frequencies of MAIT cells in the thymus, but low frequencies of MAIT cells in the periphery. After birth, MAIT cells accumulate to high frequencies in the mucosa and the spleen, but remain stable in the thymus. ${ }^{40}$ In the mouse, MAIT cells are dependent on microbial colonization for their peripheral expansion. ${ }^{14,19}$ Thus, our data support the hypothesis that microbial flora serve as stimulators for MAIT cells either by directly providing antigen or indirectly by altering the local environment. Here we demonstrate that following thymic egress, MAIT cells can adapt and speculate that this is a response to peripheral microenvironmental signals. This contrasts with iNKT cells whose abundance in the peripheral blood of humans does not change from birth to adulthood. ${ }^{41}$ Thus, MAIT cell adaptation in the periphery may differ from other innate non-classical T-cell responses.

In further support of the relationship of MAIT cells with environmental signals, we found that thymic as well as cord blood Mtb-reactive MAIT cells have a naive phenotype. These findings are similar to those from Martin et al. ${ }^{15}$ who previously showed that a proportion of $\mathrm{V} \alpha 7.2^{+} \mathrm{CD} 161^{+}$thymocytes and all cord blood cells expressed CD45RA. In sharp contrast, peripheral blood Mtb-reactive MAIT cells lost cell surface expression of CD45RA and CCR7 and were uniformly of a memory phenotype. Furthermore, the lack of detectable sjTRECs from peripheral $\mathrm{Va} 7.2^{+} \mathrm{T}$ cells confirms that these cells had undergone multiple rounds of cellular division. The combination of peripheral cell expansion and cell surface phenotypic changes is consistent with MAIT cell adaptation in response to an encounter with peripheral antigen(s).

This study demonstrates that CD8 co-receptor engagement is required for the activation of MR1-restricted CD8 ${ }^{+} \mathrm{Mtb}-$ reactive $\mathrm{V} \alpha 7.2^{+} \mathrm{T}$-cell clones. Furthermore, our ex vivo analysis of innate effector function demonstrates that virtually all the Mtb-reactive MR1-restricted V $\alpha 7.2^{+} \mathrm{T}$ cells from the thymus and cord blood express CD8. Controversy exists regarding the capacity of MR1 to interact with CD8. Riegert et al. ${ }^{16}$ have argued that the mouse and human MR1 Ig-like $\alpha 3$ domains lack all the amino-acid residues thought necessary for MHC/CD8 interactions. In contrast, Walter et al. ${ }^{42}$ have found that the deduced amino-acid sequence of the rat MR1 homologue contains the prototypic CD8 binding sites. Thus, pathogen-reactive MAIT cells appear to depend on CD8 for their activation, but this may not be the case with all MAIT cells.

As discussed above, we have consistently observed that Mtbreactive MR1-restricted $\mathrm{V} \alpha 7.2^{+} \mathrm{T}$ cells from human peripheral blood express CD8 (Gold et al. ${ }^{18}$ and data not shown). By contrast, human MAIT cells have been defined on the basis on the co-expression of $\mathrm{V} \alpha 7.2^{+}$and $\mathrm{CD} 161$, and have been found in the DN and CD8 SP populations. ${ }^{13-15,18,25}$ Furthermore, MAIT cells were originally defined by the expression of a canonical TCR comprised of the semi-invariant V $\alpha 7.2 / \mathrm{J} \alpha 33^{+}$TCR $\alpha$ chain. ${ }^{13,14}$ However, 25\% of Mtb-reactive MR1-restricted T-cell clones we sequenced express the Va7.2 TCR $\alpha$ chain in combination with J $\alpha 20$ (Gold et al. ${ }^{18}$ and data not shown). These observations suggest that pathogen-reactive MR1-restricted MAIT cells may be functionally distinct from V $\alpha 7.2 / \mathrm{CD} 161^{+}$, DN MAIT cells that produce interleukin- $17 .{ }^{25}$ At present, a role for DN MAIT cells in MR1-dependent microbial recognition remains to be defined.

Pathogen-reactive MAIT cells that share features of innate and adaptive immunity are poised to play an important role in early containment of microbial infection. Representative of innate $\mathrm{T}$ cells, pathogen-reactive MAIT cells possess intrinsic effector functions that would allow them to act early in the response to infections. Also similar to other innate cells that localize to specific tissue sites, pathogen-reactive MAIT cells are found in the lung where microbial entry frequently occurs. Pathogenreactive MAIT cells can detect microbial intracellular infection of non-professional, antigen-presenting cells such as epithelial cells, ${ }^{18}$ further supporting a role for MAIT cells in peripheral tissues. Nonetheless, in addition to these innate characteristics, MAIT cells may contribute to early pathogen control in a manner akin to memory T cells. Immunological memory is represented by increased frequencies of antigen-specific cells that display enhanced functionality after primary antigenic exposure. The mature phenotype of pathogen-reactive MAIT cells in the periphery suggests that these cells have been altered by antigenic stimulation and the increases in pathogen-reactive MAIT cells in the periphery may allow for improved microbial control. Thus, human pathogen-reactive MR1-restricted MAIT cells share qualities of both innate and adaptive immunity that suggests they have functional diversity in their ability to control infection.

\section{METHODS}

Human subjects. All tissue and blood were obtained under protocols approved by the Institutional Review Board at Oregon Health and Science University. Human thymuses were obtained from children of $<4$ months of age undergoing cardiac surgery at Doernbecher Children's Hospital in Portland, OR. The TB disease incidence in Oregon in 2009 was $2.3 / 100,000$, of which only 1 was a pediatric case (Annual Tuberculosis Report, Oregon 2009). As such, neonatal thymus donors were considered TB unexposed. Single-cell suspensions were generated as described previously. ${ }^{12}$ Umbilical cord blood was obtained from the placenta of uncomplicated term pregnancies after delivery. Within $24 \mathrm{~h}$ of collection into CPT tubes (BD Pharmingen, San Jose, CA), the blood was processed and cord blood mononuclear cells were obtained after centrifugation. Peripheral blood mononuclear cells were obtained by apheresis from healthy adult donors with informed consent. Adult subjects lacked any detectable responses to Mtb immunodominant antigens CFP-10 and ESAT- 6 and were considered TB unexposed.

Mycobacteria. The H37Rv strain of Mtb was used for all live Mtb infections (ATCC, Manassas, VA), prepared as described previously, ${ }^{43}$ and infected at multiplicity of infection of 30 , unless stated otherwise. ${ }^{44}$

Cells. A549 cells (ATCC CCL-185) were used as stimulators for direct ex vivo determination of Mtb-reactive T cells. Monocyte-derived dendritic cells were prepared as described previously. ${ }^{18}$

Generation of anti-V $\alpha 7.2$ antibody OF-5A12. Animal care and immunization procedures were performed in accordance with a protocol approved 
by the Oregon Health and Science University Institutional Animal Care and Use Committee. A modified subtractive immunization strategy ${ }^{45,46}$ was used for generation of the OF-5A12 hybridoma. Briefly, mice were injected with $6^{\mathrm{e}} 6 \mathrm{CD}^{+}{ }^{+}$T-cell clones lacking the V $\alpha 7.2 \mathrm{TCR}$. At 24 and $48 \mathrm{~h}$ after the initial immunization, mice were treated with cyclophosphamide to ablate the response to the T cells lacking Va7.2 TCR. At 3 and 6 weeks following the initial immunization, mice were injected with $2^{\mathrm{e}} 6$ $\mathrm{CD}^{+}{ }^{+}$MAIT cell clones expressing the Va7.2 TCR, from the same donor. The MAIT cell clone was confirmed to express V $\alpha 7.2$ by positive $3 \mathrm{C} 10$ staining. ${ }^{18}$ Furthermore, this clone was confirmed to be MR1 restricted by antibody blockade (not shown). The fusion of splenocytes with SP2/0 was performed at 5 days following the final boost using standard cell-cell fusion techniques. Confirmation of OF-5A12 antibody specificity was performed by flow cytometry on an extensive panel of $\mathrm{V} \alpha 7.2^{+}$- and $\mathrm{V} \alpha 7.2^{-}$-expressing cells that were also stained with a previously characterized monoclonal antibody specific for the V $\alpha 7.2$ TCR (Supplementary Figure S1 online). The specificity of OF-5A12 for the Va7.2 TCR was confirmed by blocking the ability of $3 \mathrm{C} 10$ to bind MAIT cells after OF5A12 pre-incubation (Supplementary Figure S2 online).

Isolation of $\mathbf{C D 8}^{+} \mathbf{V} \alpha \mathbf{7 . 2}^{+} \mathbf{T}$ cells. $\mathrm{CD}^{+}{ }^{+} \mathrm{CD} 4^{-} \mathrm{T}$ cells from peripheral blood mononuclear cells and cord blood were enriched by negative selection using magnetic bead separation according to the manufacturer's instructions (Miltenyi, Auburn, CA), except that for thymocytes we doubled the amount of CD4 beads due to the high frequency of $\mathrm{CD} 4^{+}$ cells in the thymus. FACS was used to isolate $\mathrm{V} \alpha 7.2^{+} \mathrm{CD}^{+}(\mathrm{SP})$ and $\mathrm{CD}^{-}{ }^{-} \mathrm{CD}^{-}{ }^{-}$(DN) cells that lacked CD4 (BioLegend, San Diego, CA) and the $\gamma \delta$ TCR (Thermo, Rockford, IL).

Intracellular cytokine staining assay. $\mathrm{CD}^{+}$and $\mathrm{DN} \mathrm{T}$ cells from peripheral blood mononuclear cells and cord blood were selected using negative selection of $\mathrm{T}$ cells and then depleted of $\mathrm{CD}^{+}{ }^{+}$cells by positive selection using magnetic bead separation according to the manufacturer's instructions (Miltenyi). CD4-depleted T cells were added to Mtb-infected or - uninfected A549 cells at ratio of 3:1 and incubated for 16h. GolgiStop (BD Pharmingen) was added for the final $6 \mathrm{~h}$ of the assay. Cells were first stained with LIVEDEAD Fixable Dead Cell Stain Kit (Invitrogen, Grand Island, NY) before being surface stained for the expression of the $\mathrm{V} \alpha 7.2$ TCR using anti-V $\alpha 7.2$ antibody (clone OF-5A12-described below and Supplementary Figures $\mathbf{S 1}$ and $\mathbf{S 2}$ online) CD8 $\alpha$, CD45RA, CCR7, and $\mathrm{CD} 62 \mathrm{~L}$, and subsequently fixed and permeabilized with Cytofix/CytoPerm (BD Pharmingen), and then stained in the presence of Perm/Wash (BD Pharmingen), with fluorochrome-conjugated antibodies to TNF- $\alpha$ (Beckman Coulter, Indianapolis, IN). Acquisition was performed with an LSRII flow cytometer with FACS Diva software (BD Pharmingen). All analyses were performed using the FlowJo software (TreeStar, Ashland, OR).

Generation and isolation of functional MAIT cells. CD4-depleted thymocytes were added to uninfected A549 cells at a ratio of 3:1 and incubated for $2 \mathrm{~h}$ in the presence of phorbol myristate acetate $\left(20 \mathrm{ng} \mathrm{ml}^{-1}\right.$; Sigma, St Louis, MO) and ionomycin ( $1 \mu \mathrm{M}$; Sigma), $0.5 \mathrm{ng} \mathrm{ml}^{-1}$ interelukin-2 and anti-CD107 $\alpha$ (LAMP)-PE $\left(10 \mu \mathrm{lml}^{-1}\right.$; BD Biosciences, San Jose, CA). Cells were then stained with fluorochrome-conjugated antibodies to CD8 $\alpha$ (BioLegend), V $\alpha 7.2$ (OF-5A12) CD4 (BioLegend), $\gamma \delta T C R$ (Thermo), and propidium iodide (BD Biosciences). $\gamma \delta \mathrm{TCR}^{-}, \mathrm{CD}^{-}$, propidium iodide ${ }^{-}, \mathrm{CD} 8^{+}$cells were sorted into four subsets (a minimum of 50,000 cells each) based on V $\alpha 7.2$ and LAMP expression using a FACS Vantage and sjTREC analysis performed as described below.

sjTREC content. sjTREC content was quantified as described by Haines et al. ${ }^{23}$ using plasmids generously provided to us by David B Lewis. Briefly, total cellular DNA was isolated using a QIAamp DNAMini kit (Qiagen, Valencia, CA) and used in $20.0 \mu \mathrm{l}$ real-time polymerase chain reaction reactions in 96-well plates analyzed in a Step One Plus RealTime PCR instrument (Applied Biosystems, Carlsbad, CA). Reactions included oligonucleotide primers and a TAMRA-FAM-labeled internal probe for the $\delta$ Rec- $\Psi \mathrm{J} \alpha$ signal joint. ${ }^{47}$ A TAMRA-FAM-labeled internal probe for the $\mathrm{C} \alpha$ region of the TCR- $\alpha$ gene locus was used for the detection of $\mathrm{C} \alpha$ copy number. Plasmids were used in standard curves ranging from $1.0 \times 10^{1}$ to $1.0 \times 10^{5}$ copies per reaction. ${ }^{23,47}$

SUPPLEMENTARY MATERIAL is linked to the online version of the paper at http://www.nature.com/mi

\section{ACKNOWLEDGMENTS}

We thank Dr Lewis for kindly providing us with plasmids and protocols to perform sjTREC analyses, Dr Hansen for his generous gift of the antiMR1 antibody, members of the Operating Room Team at Doernbecher's Children's Hospital for their collaboration in collecting thymus for our studies, Dr Segel and the members of the Labor and Delivery Ward at OHSU for their collaboration in collecting cord blood for our studies, and Erin Merrifield for her expert assistance with human subjects protocols. Funding was provided in part by the Department of Veterans Affairs and with resources and the use of facilities at the Portland VA Medical Center, National Institutes of Health (NIH) grants Al078965 and AI48090, and $\mathrm{NIH}$ contract HHSN266200400081C.

\section{DISCLOSURE}

The authors declared no conflict of interest.

() 2013 Society for Mucosal Immunology

\section{REFERENCES}

1. Orme, I.M. The kinetics of emergence and loss of mediator T lymphocytes acquired in response to infection with Mycobacterium tuberculosis. J. Immunol. 138, 293-298 (1987).

2. Kamath, A., Woodworth, J.S. \& Behar, S.M. Antigen-specific CD8+ $T$ cells and the development of central memory during Mycobacterium tuberculosis infection. J. Immunol. 177, 6361-6369 (2006).

3. Billeskov, R., Vingsbo-Lundberg, C., Andersen, P. \& Dietrich, J. Induction of CD8 T cells against a novel epitope in TB10.4: correlation with mycobacterial virulence and the presence of a functional region of difference-1. J. Immunol. 179, 3973-3981 (2007).

4. Lewinsohn, D.M. et al. High resolution radiographic and fine immunologic definition of TB disease progression in the rhesus macaque. Microbes Infect. 8, 2587-2598 (2006).

5. Reiley, W.W. et al. ESAT-6-specific CD4 T cell responses to aerosol Mycobacterium tuberculosis infection are initiated in the mediastinal lymph nodes. Proc. Natl. Acad. Sci. USA 105, 10961-10966 (2008).

6. Wolf, A.J. et al. Initiation of the adaptive immune response to Mycobacterium tuberculosis depends on antigen production in the local lymph node, not the lungs. J. Exp. Med. 205, 105-115 (2008).

7. Chiu, N.M., Wang, B., Kerksiek, K.M., Kurlander, R., Pamer, E.G. \& Wang, C.R. The selection of M3-restricted T cells is dependent on M3 expression and presentation of $\mathrm{N}$-formylated peptides in the thymus. J. Exp. Med. 190, 1869-1878 (1999).

8. Urdahl, K.B., Sun, J.C. \& Bevan, M.J. Positive selection of MHC class Ib-restricted CD8(+) T cells on hematopoietic cells. Nat. Immunol. 3, 772-779 (2002).

9. Gapin, L., Matsuda, J.L., Surh, C.D. \& Kronenberg, M. NKT cells derive from double-positive thymocytes that are positively selected by CD1d. Nat. Immunol. 2, 971-978 (2001).

10. Berzins, S.P., Cochrane, A.D., Pellicci, D.G., Smyth, M.J. \& Godfrey, D.I. Limited correlation between human thymus and blood NKT cell content revealed by an ontogeny study of paired tissue samples. Eur. J. Immunol. 35, 1399-1407 (2005).

11. Bendelac, A., Savage, P.B. \& Teyton, L. The biology of NKT cells. Annu. Rev. Immunol. 25, 297-336 (2007).

12. Gold, M.C. et al. Human innate Mycobacterium tuberculosis-reactive alphabetaTCR+ thymocytes. PLoS Pathogen. 4, e39 (2008).

13. Porcelli, S., Yockey, C.E., Brenner, M.B. \& Balk, S.P. Analysis of T cell antigen receptor (TCR) expression by human peripheral blood $\mathrm{CD}^{-}{ }^{-}{ }^{-}$ alpha/beta T cells demonstrates preferential use of several $\mathrm{V}$ beta genes and an invariant TCR alpha chain. J. Exp. Med. 178, 1-16 (1993).

14. Treiner, E. et al. Selection of evolutionarily conserved mucosal-associated invariant T cells by MR1. Nature 422, 164-169 (2003).

15. Martin, E. et al. Stepwise development of MAIT cells in mouse and human. PLoS Biol. 7, e54 (2009). 
16. Riegert, P., Wanner, V. \& Bahram, S. Genomics, isoforms, expression, and phylogeny of the MHC class I-related MR1 gene. J. Immunol. 161, 4066-4077 (1998).

17. Huang, S. et al. Evidence for MR1 antigen presentation to mucosalassociated invariant T cells. J. Biol. Chem. 280, 21183-21193 (2005).

18. Gold, M.C. et al. Human mucosal associated invariant T cells detect bacterially infected cells. PLoS Biol. 8, e1000407 (2010).

19. Le Bourhis, L. et al. Antimicrobial activity of mucosal-associated invariant T cells. Nat. Immunol. 11, 701-708 (2010).

20. Alanio, C., Lemaitre, F., Law, H.K., Hasan, M. \& Albert, M.L. Enumeration of human antigen-specific naive CD $8+T$ cells reveals conserved precursor frequencies. Blood 115, 3718-3725 (2010).

21. Hale, J.S. \& Fink, P.J. Back to the thymus: peripheral T cells come home. Immunol. Cell Biol. 87, 58-64 (2009).

22. Douek, D.C. et al. Changes in thymic function with age and during the treatment of HIV infection. Nature 396, 690-695 (1998).

23. Haines, C.J. et al. Human CD4+ T cell recent thymic emigrants are identified by protein tyrosine kinase 7 and have reduced immune function. J. Exp. Med. 206, 275-285 (2009).

24. Betts, M.R. et al. Sensitive and viable identification of antigen-specific CD8+ T cells by a flow cytometric assay for degranulation. J. Immunol. Methods 281, 65-78 (2003).

25. Dusseaux, M. et al. Human MAIT cells are xenobiotic-resistant, tissuetargeted, CD161hi IL-17-secreting T cells. Blood 117, 1250-1259 (2011).

26. Fujii, Y., Okumura, M., Inada, K., Nakahara, K. \& Matsuda, H. CD45 isoform expression during T cell development in the thymus. Eur. J. Immunol. 22, 1843-1850 (1992).

27. Fukuhara, K. et al. A study on CD45 isoform expression during T-cell development and selection events in the human thymus. Hum. Immunol. 63, 394-404 (2002).

28. Spits, $\mathrm{H}$. Development of alphabeta T cells in the human thymus. Nat Rev. Immunol. 2, 760-772 (2002).

29. Campbell, J.J., Pan, J. \& Butcher, E.C. Cutting edge: developmental switches in chemokine responses during T cell maturation. J. Immunol. 163, 2353-2357 (1999).

30. Holder, J.E., Kimpton, W.G., Washington, E.A. \& Cahill, R.N. L-selectin expression on thymic emigrants defines two distinct tissue-migration pathways. Immunology 98, 422-426 (1999).

31. Cho, H., Bediako, Y., Xu, H., Choi, H.J. \& Wang, C.R. Positive selecting cell type determines the phenotype of MHC class Ib-restricted CD8+ T cells. Proc. Natl. Acad. Sci. USA 108, 13241-13246 (2011).

32. Hashimoto, K., Hirai, M. \& Kurosawa, Y. A gene outside the human $\mathrm{MHC}$ related to classical HLA class I genes. Science 269, 693-695 (1995).
33. Chua, W.J. et al. Endogenous MHC-related protein 1 is transiently expressed on the plasma membrane in a conformation that activates mucosal-associated invariant T cells. J. Immunol. 186, 4744-4750 (2011).

34. Coles, M.C. \& Raulet, D.H. NK1.1 ${ }^{+}$T cells in the liver arise in the thymus and are selected by interactions with class I molecules on $\mathrm{CD}^{+}{ }^{+} \mathrm{CD} 8^{+}$ cells. J. Immunol. 164, 2412-2418 (2000).

35. Georgel, P., Radosavljevic, M., Macquin, C. \& Bahram, S. The nonconventional MHC class I MR1 molecule controls infection by Klebsiella pneumoniae in mice. Mol. Immunol. 48, 769-775 (2011).

36. Gozalbo-Lopez, B. et al. The MHC-related protein 1 (MR1) is expressed by a subpopulation of CD38+, IgA+ cells in the human intestinal mucosa. Histol. Histopathol. 24, 1439-1449 (2009).

37. Kovalovsky, D. et al. The BTB-zinc finger transcriptional regulator PLZF controls the development of invariant natural killer $\mathrm{T}$ cell effector functions. Nat. Immunol. 9, 1055-1064 (2008).

38. Savage, A.K. et al. The transcription factor PLZF directs the effector program of the NKT cell lineage. Immunity 29, 391-403 (2008).

39. Thomas, S.Y. et al. PLZF induces an intravascular surveillance program mediated by long-lived LFA-1-ICAM-1 interactions. J. Exp. Med. 208 1179-1188 (2011)

40. Goldfinch, N., Reinink, P., Connelley, T., Koets, A., Morrison, I. \& Van Rhijn, I. Conservation of mucosal associated invariant T (MAIT) cells and the MR1 restriction element in ruminants, and abundance of MAIT cells in spleen. Vet. Res. 41, 62 (2010).

41. Bienemann, K. et al. iNKT cell frequency in peripheral blood of Caucasian children and adolescent: the absolute iNKT cell count is stable from birth to adulthood. Scand J. Immunol. 74, 406-411 (2011).

42. Walter, L. \& Gunther, E. Isolation and molecular characterization of the rat MR1 homologue, a non-MHC-linked class I-related gene. Immunogenetics 47, 477-482 (1998).

43. Lewinsohn, D.M. et al. Classically restricted human CD8+ T lymphocytes derived from Mycobacterium tuberculosis-infected cells: definition of antigenic specificity. J. Immunol. 166, 439-446 (2001).

44. Lewinsohn, D.A., Heinzel, A.S., Gardner, J.M., Zhu, L., Alderson, M.R. \& Lewinsohn, D.M. Mycobacterium tuberculosis-specific CD8+ T cells preferentially recognize heavily infected cells. Am. J. Respir. Crit. Care Med. 168, 1346-1352 (2003).

45. Sleister, H.M. \& Rao, A.G. Strategies to generate antibodies capable of distinguishing between proteins with $>90 \%$ amino acid identity. J. Immunol. Methods 252, 121-129 (2001).

46. Williams, C.V., Stechmann, C.L. \& McLoon, S.C. Subtractive immunization techniques for the production of monoclonal antibodies to rare antigens. Biotechniques 12, 842-847 (1992).

47. Hazenberg, M.D., Verschuren, M.C., Hamann, D., Miedema, F. \& van Dongen, J.J. T cell receptor excision circles as markers for recent thymic emigrants: basic aspects, technical approach, and guidelines for interpretation. J. Mol. Med. 79, 631-640 (2001). 